\title{
Évolution des productions animales terrestres et aquacoles dans le monde : tendances globales et implications économiques, sociales et environnementales
}

\author{
P. GERBER', C. BRUGÈRE', P. ANKERS \\ ${ }^{1}$ FAO-OAA, Organisation de l'Alimentation et de l'Agriculture des Nations Unies, \\ Division de la Production et de la Santé Animale, Rome, Italie \\ 2 FAO-OAA, Organisation de l'Alimentation et de l'Agriculture des Nations Unies, \\ Division de l'Utilisation et de la Conservation des Ressources de Pêches \\ et de l'Aquaculture, Rome, Italie \\ Courriel :Pierre.Gerber@fao.org
}

L'élevage et l'aquaculture constituent le secteur le plus dynamique de l'agriculture et des pêches. Cet article présente une revue comparée des tendances mondiales de ces deux formes de production animale.

Le secteur des productions animales, entendu ici comme regroupant l'élevage et l'aquaculture, constitue la part la plus dynamique de l'agriculture et des pêches. Il se caractérise généralement par une forte croissance de la production ainsi que des transformations profondes des filières et des techniques de production. Ces tendances ont été décrites dans de récentes publications (FAO 2009a et b, Gerber et al 2010, Steinfeld et al 2010), mais de manière séparée pour l'élevage et l'aquaculture. Bien que différentes, ces deux activités sont liées, au niveau de la substitution de leurs produits sur le marché, de l'utilisation des ressources naturelles et de leur rôle dans la sécurité alimentaire et le développement rural. Cet article propose une revue comparée des tendances mondiales concernant ces deux formes de production animale.

La première section de cet article traite de la croissance des productions animales et de ses moteurs. Les transformations des techniques de production et des filières, ainsi que les impacts sociaux, économiques et environnementaux de ces transformations, sont décrites dans les deux sections qui suivent. La dernière section propose une synthèse et des perspectives quant aux enjeux communs de l'élevage et de l'aquaculture. Bien que brossant un tableau global des productions animales, la discussion des enjeux du secteur met l'accent sur les pays dits en développement, où la croissance est la plus forte.

\section{1 / Productions en forte croissance}

\section{1 / Tendances et facteurs moteurs de la consommation}

Le secteur de l'élevage, tout comme ceux de l'alimentation et de l'agriculture en général, connaît des changements considérables dont bon nombre sont le résultat de facteurs externes. L'augmentation des populations et d'autres paramètres démographiques tels que la répartition par âge et l'urbanisation déterminent la demande alimentaire ; c'est toutefois la croissance écono- mique et l'augmentation des revenus individuels qui contribue de manière prépondérante à l'augmentation de la demande en produits animaux. On constate en effet une grande élasticité de la demande en produits animaux par rapport aux revenus (Dey et al 2008, FAO 2009 b) ; en d'autres termes, l'augmentation des revenus provoque une augmentation rapide des dépenses en produits d'élevage. Le lien entre le revenu par habitant et la consommation de viande en 2005 est illustré par la figure 1. Le graphique montre l'effet positif de la hausse des revenus sur la consommation des produits de l'élevage, en particulier dans les pays à faible revenu.

Figure 1. PIB et consommation de viande par habitant et par pays (2005) (Source : FAO 2009b).

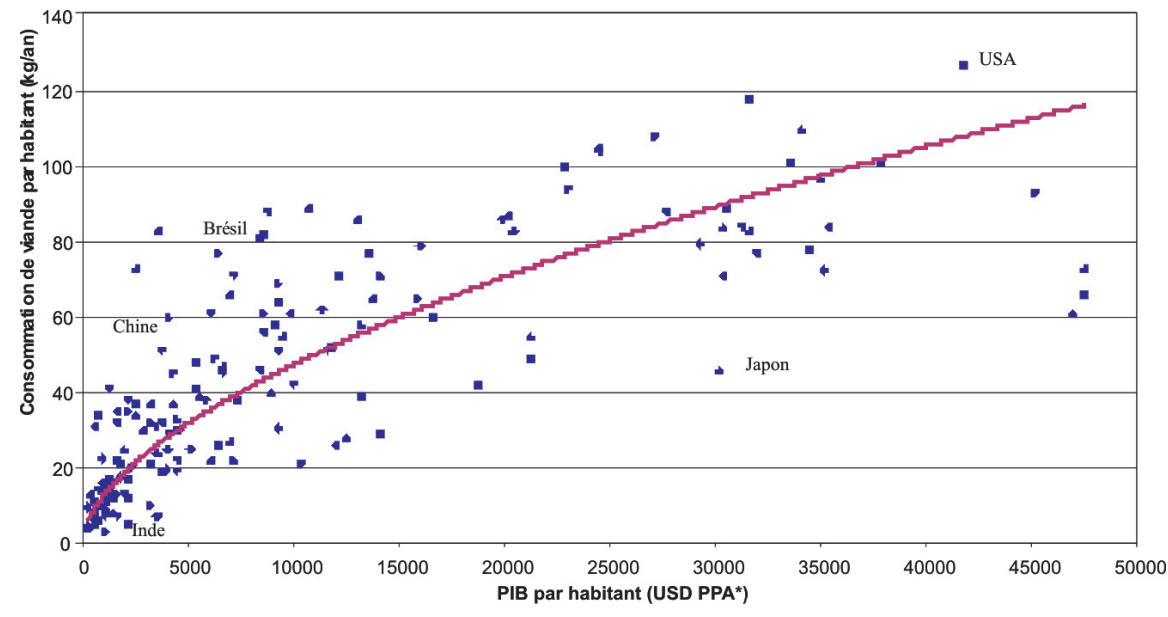

* Le PIB par habitant est mesuré en Parité du Pouvoir d'Achat (PPA) en dollars US internationaux constants de 2005 
Ces tendances se sont accélérées au cours des 20 dernières années dans les régions d'Asie de l'Est et du Sud-Est, d'Amérique latine et du Proche-Orient, entraînant un accroissement rapide de la demande en produits d'origine animale et autres denrées de valeur telles que les légumes et les huiles. La Banque mondiale (2010) prévoit que la croissance moyenne des pays en développement s'accélèrera au cours des prochaines décennies; cette hausse des revenus devrait continuer à se traduire par une demande accrue en produits animaux.

\section{2 / Principales espèces et zones de production}

\section{a) Élevage}

La géographie de la production suit en grande partie celle de la consommation. Entre 1961 et 2007, la croissance la plus rapide de la production de viande a eu lieu en Asie de l'Est et du SudEst, suivie de l'Amérique latine et des Caraïbes. Le développement le plus soutenu de la production d'oeufs a été observé en Asie de l'Est et du Sud-Est, tandis que la production de lait est dominée par l'Asie du Sud. En 2007, les pays en développement avaient dépassé les pays développés en ce qui concerne la production de viande (tableau 1) et d'œufs et comblaient leur retard pour ce qui est de la production de lait.

Trois pays dominent le secteur de 1 'élevage en termes de volumes produits : la Chine, Le Brésil et l'Inde. La Chine et le Brésil affichent les plus fortes croissances à la production, notamment pour la viande. Entre 1980 et 2007, la Chine a multiplié sa production de viande par six. Elle représente aujourd'hui près de $50 \%$ de la production de viande des pays en développement et $31 \%$ de la production mondiale. Le Brésil a multiplié sa production de viande quasiment par quatre et produit $11 \%$ de la production de viande des pays en développement et $7 \%$ de la production mondiale.

Dans les autres parties du monde en développement, la croissance de la production de viande - ainsi que les niveaux de production - a été plus faible. Bien qu'elle ait plus que doublé entre 1980 et 2007, la production de viande en Inde reste peu élevée. Cependant, l'Inde compte désormais pour près de $15 \%$ de la production mondiale de lait (la production y a été plus que triplée entre 1980 et 2007). La production de viande, lait et oeufs a également progressé en Afrique subsaharienne mais plus lentement que dans d'autres régions (FAO 2009b).

La hausse de la production concerne particulièrement les espèces monogas-

Tableau 1. Production (en millions de Tonnes) des principales catégories de viande par région/pays en 2007.

\begin{tabular}{lcccc}
\hline \multicolumn{1}{c}{ Région/groupe de pays } & Porc & Volailles & Bovins & $\begin{array}{c}\text { Moutons } \\
\text { et chèvres }\end{array}$ \\
\hline Pays développés & 39,5 & 37,0 & 29,4 & 3,2 \\
\hline Pays en développement & 76,0 & 49,8 & 32,5 & 10,8 \\
$\quad$ Asie de l'Est et du Sud-Est & 8,3 & 6,8 & 1,5 & 0,4 \\
$\quad$ hors Chine & 60,0 & 15,3 & 7,3 & 4,9 \\
Chine & 6,1 & 17,2 & 15,8 & 0,5 \\
Amérique Latine et Caraïbes & 0,5 & 3,0 & 2,1 & 1,5 \\
Asie du Sud & 0,1 & 5,3 & 1,8 & 2,0 \\
Proche-Orient & 0,8 & 2,0 & 4,0 & 1,6 \\
$\quad$ et Afrique du Nord & 115,5 & 86,8 & 61,9 & 14,0 \\
$\quad$ Afrique subsaharienne & & &
\end{tabular}

Source : FAO 2009b.

triques; la production de viande de volaille est le sous-secteur qui s'est développé le plus rapidement, suivi par la production de porc. L'augmentation de la production de viande de petits et grands ruminants a été beaucoup plus modeste. La composition de la production mondiale de viande a donc subi des modifications profondes, et les différences entre les régions et les pays sont importantes (tableau 1).

La viande de porc représente plus de $40 \%$ des disponibilités mondiales de viande, en partie à cause des niveaux de production élevés et de la croissance rapide observés en Chine, qui génère plus de la moitié de la production mondiale. La production de viande de volaille qui, en 2007 , représentait plus de $26 \%$ des disponibilités mondiales de viande, a été plus homogènement répartie entre les pays développés et les pays en développement. Au niveau mondial, la production de viande bovine a beaucoup moins augmenté et uniquement dans les pays en développement. La viande de petits ruminants reste d'une importance mineure au niveau mondial, mais elle représente une proportion importante de la viande produite au Proche-Orient et en Afrique du Nord, en Afrique subsaharienne et en Asie du Sud.

Aujourd'hui, l'Afrique a un rôle très réduit dans le volume d'exportations en viandes et animaux vivants (Rich 2009). Elle ne fournit que $1 \%$ de l'ensemble des exportations pour le bœuf, le porc ou la volaille et cette part n'évolue pas. Pour les exportations de bœuf, de volaille et de chasse, la très grande majorité vient des pays d'Afrique australe notamment l'Afrique du Sud, la Namibie et le Botswana. La viande de chèvre et de porc vient essentiellement d'Afrique de l'Est alors que les exporta- tions de viande de mouton viennent d'abord d'Afrique du Nord (principalement du Soudan).

\section{b) Aquaculture}

La production aquacole mondiale s'est considérablement accrue au cours des 50 dernières années et l'aquaculture représentait $47 \%$ de l'offre mondiale de poisson en 2006. La même année, en Chine, $90 \%$ de la production de poisson à des fins alimentaires provenaient de l'aquaculture (FAO 2007). L'aquaculture poursuit son essor à un rythme plus rapide que celui de tous les autres secteurs de production alimentaire d'origine animale, avec un taux de croissance moyen annuel mondial se maintenant à $8,7 \%$ (sauf pour la Chine avec 6,5\%) depuis 1970 (figure 2). En 2008, le volume total de produits aquacoles était de 68 millions de Tonnes, contre 104 pour la viande de porc, 90 pour la viande de volaille, 62 pour la viande bovine, 13 pour la viande de petits ruminants et 694 pour le lait (FAOSTAT 2010).

La production est essentiellement issue d'Asie: en 2006, les pays de la région Asie-Pacifique représentaient $89 \%$ de la production mondiale en volume (tableau 2) et $77 \%$ de sa valeur. La Chine seule représenterait $67 \%$ du volume total de la production aquacole mondiale, et $49 \%$ de sa valeur. Une analyse de la production par région pour la période 19702006 montre cependant que la croissance n'a pas été uniforme : alors que les taux de croissance annuels depuis 1970 sont positifs pour la région Amérique latine et Caraïbes (22\%), la région Proche-Orient (20\%) et la région Afrique (12,7\%), ils ont chuté en Chine, en Europe et en Amérique du Nord depuis les années 90 et début des années 2000, reflétant la certaine maturité que le sous-secteur a atteint dans ces régions. 
Figure 2. Evolution comparée des principales productions animales entre 1988 et 2008 (volumes de production indexés sur la valeur en 1988).

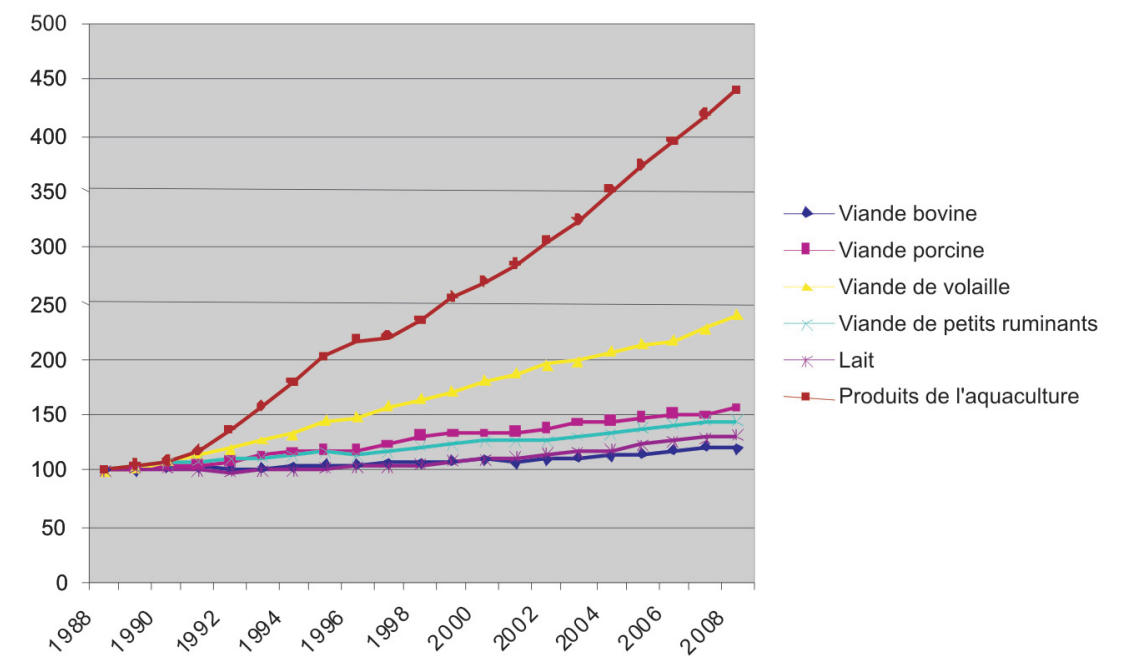

Source : FAOSTAT 2010

La production en eaux douces représente $58 \%$ en volume et $48 \%$ en valeur, alors que l'aquaculture dans les eaux marines représente $34 \%$ des quantités produites et $36 \%$ de la valeur totale. La production marine est principalement orientée vers les poissons à forte valeur, mais on compte également un volume important de moules et d'huîtres. La production en eau saumâtre ne représentait que $8 \%$ des quantités produites en 2006, mais sa part s'établissait à $16 \%$ de la valeur totale, ce qui s'explique par la place prépondérante des crustacés à valeur commerciale élevée.

Concernant les espèces élevées, la production aquacole de la Chine, de l'Asie du Sud et de la majeure partie de l'Asie du Sud-Est est principalement composée de carpes (cyprinidés), tandis que dans le reste de l'Asie orientale, elle se compose essentiellement de poissons marins de valeur. La région AsiePacifique représente $98 \%$ des carpes (dont $77 \%$ sont produites en Chine et consommées principalement en Asie) et
95\% des huîtres produites $(82 \%$ en Chine). Le second groupe le plus produit après celui des carpes est celui des tilapias avec 2,5 millions de Tonnes (FAO 2010c). Ce groupe fait l'objet d'élevage dans plus de 100 pays et est consommé dans le monde entier. La croissance de la production de poissonchat «panga» (Pangasius spp.), en particulier au Vietnam, a été spectaculaire au cours des dernières années, croissant à un taux annuel moyen de $24,9 \%$ depuis 2003 pour atteindre 1,2 million de Tonnes produites en 2008 , dont plus de la moitié sont exportées sous forme de filets, vers l'Europe surtout (Globefish 2009). 98\% des crevettes et bouquets (pénéides) proviennent aussi de cette région, les cinq plus gros producteurs (Chine, Thaillande, Vietnam, Indonésie et Inde) comptant pour $81 \%$ de la production.

Dans la région Amérique latine et Caraïbes, les salmonidés ont évincé les crevettes durant la dernière décennie pour devenir le premier groupe d'espè-

Tableau 2. Productions animales (en volume) par région/pays en 2006.

\begin{tabular}{lcccc}
\hline \multicolumn{1}{c}{ Région/Pays } & $\begin{array}{c}\text { Aquaculture } \\
(\%)\end{array}$ & $\begin{array}{c}\text { Viande } \\
(\%)\end{array}$ & $\begin{array}{c}\text { Lait } \\
(\%)\end{array}$ & $\begin{array}{c}\text { CEufs } \\
(\%)\end{array}$ \\
\hline Asie et Pacifique (hors Chine) & 22,8 & 13,7 & 29,5 & 18,5 \\
Chine & 66,7 & 26,9 & 5,4 & 39,9 \\
Europe & 4,2 & 19,9 & 32,4 & 16,0 \\
Amérique Latine et Caraïbes & 3,0 & 15,5 & 10,6 & 10,0 \\
Afrique & 1,5 & 5,1 & 5,2 & 3,8 \\
Amérique du nord & 1,2 & 17,0 & 13,6 & 9,2 \\
Proche-Orient & 0,6 & 1,9 & 3,3 & 2.5 \\
\hline
\end{tabular}

Source : FAO 2009a, FAOSTAT 2010. ces cultivées suite aux flambées de maladies dans les principales zones productrices de crevettes et grâce à la croissance rapide de la production de saumon au Chili. En Amérique du Nord, la barbue d'Amérique est la première espèce aquacole cultivée aux Etats-Unis d'Amérique, tandis que le saumon de l'Atlantique et le saumon du Pacifique prédominent au Canada. Par ailleurs, le Chili et la Norvège sont les premiers producteurs de saumons d'élevage (salmonidés), représentant respectivement 33 et $31 \%$ de la production mondiale.

L'Afrique subsaharienne, malgré son potentiel naturel, continue de produire peu par rapport aux autres régions. Le Nigeria est en tête, avec une production déclarée de 85000 Tonnes de poissonchat, de tilapia et autres poissons d'eau douce. Plusieurs signes encourageants sont à signaler sur le continent : la production de crevettes géantes tigrées (Penaeus monodon) à Madagascar et de l'algue Eucheuma en République-Unie de Tanzanie est en plein essor, tandis que la production d'espèces occupant des créneaux particuliers comme les ormeaux (Haliotis spp.) en Afrique du Sud-Est est elle aussi en augmentation. En Afrique du Nord, l'Egypte est de loin le premier pays en termes de production (avec 99\% du total régional), et est désormais le deuxième producteur de tilapia après la Chine et le premier producteur mondial de mulets.

\section{2 / Transformation de la production et de la com- mercialisation}

Les systèmes d'élevage ont profondément changé au cours des cinquante dernières années, évoluant d'une production dite «traditionnelle» à petite échelle vers une production industrielle qui vise les économies d'échelle et intègre souvent les différentes étapes de la production, et parfois même de la commercialisation (génétique, préparation de l'aliment, abattage et transformation). Cette transformation va de pair avec la rapide croissance du secteur qu'elle rend possible, et est donc particulièrement visible là où la croissance est forte (notamment Asie de l'Est et du Sud-Est et Amérique latine) ainsi que pour les sous-secteurs qui ont connu la croissance la plus accentuée (notamment la volaille et les oeufs, le porc et les produits laitiers). Les systèmes de production aquacoles sont, de manière générale, plus variés, moins mécanisés et moins utilisateurs de technologies avancées que l'élevage : les petits producteurs y côtoient souvent des entreprises internationales. Des transformations technologiques récentes, ainsi que 
l'adoption de codes de conduites et de meilleures pratiques de gestion des unités de production ont toutefois également permis au secteur aquacole de réaliser d'importants gains de productivité et une meilleure qualité des produits finaux.

\section{1 / Progrès technologiques}

Concernant l'élevage, la diffusion de technologie moderne en matière de génétique, de reproduction et d'alimentation a engendré une amélioration générale de la productivité. L'application de meilleures techniques d'irrigation et de fertilisation dans les cultures, conjuguée à l'utilisation de variétés améliorées et à la mécanisation, continue à accroître les rendements et à améliorer la composition et la digestibilité des pâtures et principales cultures fourragères (aliments concentrés inclus). Par ailleurs, l'utilisation de technologies modernes d'information et autres innovations techniques et institutionnelles (transport, commerce, assurances...) permettent d'améliorer les opérations après récolte ainsi que la distribution et la commercialisation des produits d'origine animale. En aquaculture, des avancées technologiques telles que les cages automatisées offshore, les systèmes recirculants, l'amélioration des stocks et une meilleure gestion génétique et sanitaire permettent le développement continu du secteur (Bostock et al 2010). De manière générale, ces changements ont permis d'augmenter l'efficience des systèmes de production animale, du moins concernant l'utilisation de ressources pour lesquelles des droits d'accès ou de propriété ainsi qu'un marché existent.

\section{a) Génétique}

Les progrès réalisés dans le domaine de la sélection génétique, notamment pour une utilisation plus efficace de l'aliment, ont été importants dans le domaine de l'élevage. L'insémination artificielle et le transfert d'embryons sont des techniques qui ont permis une rapide diffusion de matériel génétique amélioré. Ces technologies, couramment utilisées dans les pays développés sont de plus en plus diffusées dans les pays en voie de développement.

Dans le cas de l'aquaculture, les progrès faits en matière de reproduction sélective ont également été remarquables et ont permis des taux de croissance supérieurs à 10\% par génération (World Bank 2007). Ces développements demeurent cependant hors de portée des petits producteurs par manque de formation et de connaissances (FAO 2009a).
Les progrès réalisés en génétique moléculaire ont également permis le développement de nouvelles approches en élevage, telles que la sélection génomique pour la lutte contre les maladies et l'élimination des défauts génétiques. Des techniques plus récentes comme le clonage ou la transgenèse (intégration d'un ou plusieurs gènes dans un organisme vivant) pourraient avoir des impacts positifs à l'avenir, mais le cadre scientifique, politique, économique et institutionnel nécessaire à une utilisation durable et équitable de ces technologies n'est souvent pas encore en place (FAO 2008).

\section{b) Alimentation}

L'élevage a traditionnellement permis de tirer profit de ressources localement disponibles, telles que les résidus de culture et les pâturages, sans valeur nutritive pour l'Homme. Lorsque le secteur croît et s'intensifie, ces ressources locales perdent en importance, en faveur d'aliments concentrés provenant des marchés nationaux et internationaux. Ainsi, l'utilisation d'aliments concentrés pour animaux a plus que doublé dans les pays en développement entre 1980 et 2005 (FAO 2006).

En 2005, 742 millions de Tonnes de céréales ont été absorbées par les productions animales à l'échelle mondiale, soit près d'un tiers de la récolte mondiale de céréales et une part encore plus grande de céréales secondaires (FAO 2009b). En outre, 350 millions de Tonnes de sous-produits de la transformation riches en protéines servent d'aliments pour le bétail et la pisciculture chaque année (principalement du son, des tourteaux d'oléagineux et des farines de poissons). Les deux sous-secteurs ont une dépendance égale en farines de poisson (50\% chacun), bien que l'utilisation des huiles de poisson soit quasiment réservée à l'aquaculture (88\%) (Pike 2005). La dépendance de ces dernières de la pêche de capture ris- querait de contraindre à terme la croissance du secteur, mais la recherche de substituts aux huiles de poisson (et en particulier aux acides gras omega-3) à partir de plantes terrestres et autres sources semble prometteuse (Naylor et al 2009).

Le type d'aliment utilisé pour l'élevage des monogastriques (porcins et volailles) est relativement similaire à celui utilisé pour la production de poissons omnivores (par exemple tilapia et carpes). Les efforts faits dans le domaine de l'aquaculture pour réduire l'utilisation de farines et d'huiles de poisson contribuent à réduire encore ces différences (tableau 3 ).

L'aquaculture démontre en général une meilleure efficience concernant la conversion d'aliment concentré. Ceci est lié à la biologie des espèces ainsi qu'au milieu dans lequel elles évoluent, notamment à l'absence de régulation thermique et à un squelette relativement moins développé. La diversité des systèmes de production aquacoles, se basant sur des espèces variées et opérant à différents niveaux trophiques, permet également d'exploiter des niches moins demandeuses en ressources et plus durables. Ceci est le cas par exemple des systèmes de production intégrés, de la culture de bivalves et d'algues dont le plein potentiel de développement n'est pas encore atteint (Bostock et al 2010), ainsi que du développement de l'aquaculture en cages en pleine mer (Rubino 2008, FAO 2010b) offrant la possibilité de repousser les «frontières» de production et de maintenir, voire augmenter, les quantités produites d'espèces aquatiques. La recherche continue dans le domaine de la domestication des poissons permet en outre de capitaliser sur les interactions aquaculture/pêche de capture pour la remise en valeur et le maintien de la productivité de certains écosystèmes aquatiques, malgré la présence inévitable de risques écologiques et génétiques sur les populations

Tableau 3. Compositions relatives des aliments utilisés pour la production d'espèces monogastriques (élevage) et omnivores (aquaculture).

\begin{tabular}{lcccc}
\hline Produit & $\begin{array}{c}\text { Volailles } \\
(\%)\end{array}$ & $\begin{array}{c}\text { Porcin } \\
(\%)\end{array}$ & $\begin{array}{c}\text { Tilapia } \\
(\%)\end{array}$ & $\begin{array}{c}\text { Carpes } \\
(\%)\end{array}$ \\
\hline Céréales & $30-70$ & $45-70$ & $6-22$ & $7-19$ \\
Tourteaux oléagineux (soja, colza, etc.) & $22-35$ & $11-28$ & $35-45$ & $45-70$ \\
Protéagineux & $0-10$ & $0-19$ & & \\
Autres produits d'origine végétale & $6-35$ & $3-25$ & $30-35$ & $4-10$ \\
(son, manioc, etc.) & - & & $0-4$ & - \\
Huiles végétales & $0-6$ & $0-3$ & $4-12$ & $5-25$ \\
Farines de poisson & & & & \\
\hline
\end{tabular}

Source : FAO 2006, Rana et al 2009. 
natives. Le potentiel de développement de cette forme d'élevage aquatique «hybride», aujourd'hui mise en place dans 27 pays et concernant 80 espèces pour une production estimée à 2 millions de Tonnes de produits de la pêche, est incontesté (Bostock et al 2010).

Dans ce contexte, la particularité des espèces ruminantes est de pouvoir se nourrir entièrement de ressources végétales sans valeur nutritive pour l'Homme. Ceci permet d'élargir la base trophique des sociétés humaines, notamment dans des contextes ne permettant pas à l'agriculture de se développer. Les systèmes pastoraux peuvent ainsi être comparés à la pêche de capture.

\section{c) Santé animale}

Des progrès considérables ont aussi été faits dans le domaine de la santé animale. Dans le secteur industriel, le renforcement, la standardisation et la généralisation des mesures d'hygiène et de biosécurité (définie comme l'ensemble des mesures visant à réduire le risque d'introduction et de propagation d'organismes pathogènes dans les exploitations) ont largement contribué à contrôler les maladies. Par exemple, dans les quelques pays où l'Influenza Aviaire hautement pathogène est maintenant endémique, la FAO favorise le développement de stratégies basées non plus seulement sur la surveillance et le contrôle des foyers infectieux mais donnant aussi une large priorité aux mesures visant à limiter la circulation du virus, et ce, tout au long des filières. La mise en place de mesures de biosécurité a aussi fait l'objet d'une attention croissante dans le domaine de l'aquaculture au cours des dix dernières années, toutes espèces confondues. Ces mesures sont notamment inspirées de celles employées en aviculture et se basent sur un nombre de stratégies de gestion visant à minimiser la présence de pathogènes et sur des politiques et recommandations acceptées internationalement. Les avantages économiques de leur adoption ne sont cependant pas toujours évidents pour les petits producteurs qui voient en elles des coûts additionnels, voire superflus, de gestion, ce bien sûr tant que les maladies ne se déclarent pas (Lee 2005).

Dans les pays où l'élevage extensif ou mixte est largement pratiqué, des programmes de contrôle, voire d'éradication des maladies animales ont aussi permis de diminuer les pertes et d'augmenter l'offre de produits d'origine animale sur le marché. L'amélioration des réseaux routiers et des chaînes du froid ont rendu médicaments et vaccins vété- rinaires plus accessibles, même si la privatisation des services vétérinaires dans des zones ou le pouvoir d'achat est faible constitue parfois un frein au contrôle des maladies animales. Un bon exemple d'un tel programme est le Programme mondial d'Eradication de la Peste Bovine. Cette maladie sera officiellement déclarée éradiquée d'ici fin 2011, résultat d'une coordination internationale sans précédent.

\section{2 / Commerce et restructura- tion des filières}

Plus de la moitié de la population mondiale vit aujourd'hui en milieu urbain. Les filières d'approvisionnement se sont adaptées à cette modification des lieux et habitudes de consommation, notamment par l'émergence de nouvelles structures de commercialisation et une dépendance accrue des transports. Les progrès faits dans les domaines des transports et des communications ont en effet permis aux différents maillons des filières de se relocaliser tout en restant connectés. Souvent, les centres de production animale se sont éloignés des lieux de production des aliments et se sont rapprochés des consommateurs.

\section{a) Commerce}

La production de l'élevage est généralement destinée à la consommation nationale. Toutefois, les produits d'origine animale sont de plus en plus commercialisés à l'échelle internationale et une part accrue (10 à 15\%) de la production mondiale entre aujourd'hui sur le marché (FAO 2009b). Cette tendance a été particulièrement dynamique pour la viande de volaille. C'est toutefois l'aliment du bétail, plus que les produits de l'élevage, qui fait l'objet de commerce international, et notamment le soja : 24 à $25 \%$ de sa production était commercialisée au niveau international en 2005 (FAO 2006).

Le commerce du poisson et des produits dérivés est en revanche très actif et se caractérise par le large éventail de types de produits et de participants. Plus de $37 \%$ (équivalent poids vif) de la production font l'objet d'échanges internationaux, sous forme de produits d'alimentation humaine et animale (FAO 2009 b). En 2006, 75\% (en valeur) des exportations halieutiques des pays en développement étaient destinées aux pays développés, dont une partie en produits transformés préparés à partir de poisson importé (FAO 2009a). Les échanges entre pays en développement sont, à l'heure actuelle, limités, à cause des droits de douane élevés sur tous les produits importés appliqués par ces pays, essentiellement pour créer des recettes dont les gouvernements ont besoin. Malgré le nombre important de disputes entre pays importateurs et exportateurs, le développement et la promotion d'écolabels permettant de certifier un nombre croissant d'espèces cultivées (les dernières en date concernant la conchyliculture et la pangaculture, ASC-WWF 2010) devraient contribuer à améliorer l'acceptabilité de certains produits et rassurer les consommateurs.

\section{b) Intégration verticale}

Que ce soit dans les pays industrialisés ou en voie d'industrialisation, où le secteur de l'élevage est en pleine expansion, un nombre limité de grands groupes multinationaux jouent un rôle croissant dans les filières d'élevage. Leur force réside en particulier dans leur capacité à tirer profit des économies d'échelle. En maîtrisant un nombre accru d'échelons le long de la filière, ces groupes sont également capables de mieux résister à la volatilité des prix et de contrôler la qualité et l'hygiène des produits (Costales et al 2006).

La consolidation du secteur de l'aquaculture est un phénomène plus récent, ayant principalement lieu dans les pays développés et concernant surtout les espèces les plus commercialisées internationalement comme le saumon et, dans une moindre mesure, la crevette. Au cours des dix dernières années, l'industrie de l'élevage de saumon a vécu une période de consolidation importante durant laquelle le nombre d'entreprises produisant $80 \%$ du saumon de l'Atlantique a été réduit de 44\%, pour arriver à 51 en 2009 (Marine Harvest 2010). Selon des estimations de l'industrie des salmonidés, $40 \%$ de la production globale de saumon d'élevage en 2006 était contrôlée par 3 entreprises norvégiennes et une entreprise chilienne (Knapp et al 2007). L'industrie du saumon au Chili est à l'heure actuelle en plein processus de consolidation (FAO 2010a).

Les opinions restent divisées concernant les avantages de la consolidation et de l'intégration verticale dont le secteur de la salmoniculture a fait l'objet dans les années 90. Pour certains (Forster 2002), le processus de consolidation a permis à l'industrie norvégienne de maintenir sa compétitivité sur le marché global actuel. Pour d'autres en revanche (World Bank 2007), la faculté d'adaptation rapide des opérations encore gérées par les propriétaires individuels norvégiens leur a permis de faire face plus facilement aux contraintes de développement du secteur (à la fois en termes techniques et économiques). Des expériences similaires en Asie sont aussi citées pour démontrer que les petits 
producteurs aquacoles, une fois bien organisés et bien informés, et bien appuyés par des politiques progressives de développement rural, peuvent continuer à se développer, malgré les défis que la mondialisation et les conditions changeantes des marchés leur imposent (World Bank 2007).

Nombre de grandes entreprises intégrées ont démarré dans le domaine de l'alimentation animale (monogastriques) puis se sont diversifiées pour inclure la provende de poisson, le plus souvent en développant en parallèle leurs propres unités de production aquacole. C'est le cas par exemple de Charoen Pokphand (CP) Group en Thaillande (crevette, devenu leader dans le domaine des agroindustries) et de Jamaican Broilers Group en Jamaïque (tilapia). Ce dernier a encouragé la création de fermes «enfants», en leur garantissant les intrants et les marchés et en leur fournissant le support et l'expertise techniques requis (Hishamunda et Ridler 2002). Une situation semblable est aussi rencontrée en Indonésie où des partenariats entre petits et grands producteurs ont été établis et ont permis d'accroître les investissements dans le secteur, sous la supervision du gouvernement (Nurdjana 1999).

La pénétration des supermarchés dans les pays en développement est un phénomène relativement récent qui s'est effectué en vagues successives depuis le début des années 90, et qui a un effet important sur les filières d'élevage notamment par rapport à l'exclusion des petits producteurs (Costales et al 2006). Si les marchés restent le moyen privilégié de vente de poisson à des fins de consommation locale, l'essor de grandes chaînes de magasins et de restauration tend à déplacer le pouvoir de négociation vers les derniers stades de la chaîne de valeur, avec, par exemple, l'instauration de normes et labels privés (FAO 2009a).

\section{3 / Conséquences au niveau des sociétés et des écosystè- mes, et options pour l'amé- lioration des systèmes}

\section{1 / Impacts économiques et sociaux}

L'augmentation actuelle de la demande en produits d'origine animale est une opportunité à saisir pour les pays producteurs. Une question importante en termes de développement est de savoir quels producteurs pourront bénéficier de cette opportunité sur les marchés domestiques mais aussi régionaux et internationaux.
Dans de nombreuses régions du monde, on observe une augmentation rapide de la taille moyenne des unités de production d'élevage, parallèlement à une diminution importante du nombre d'éleveurs (FAO 2009b). Les éléments moteurs de ce phénomène sont les économies d'échelle et l'accès aux marchés. La diversité des produits et la variété des étapes du processus de production offrent un potentiel différent pour les économies d'échelle : il tend à être élevé dans les secteurs se prêtant à la mécanisation (abattoirs, laiteries, production de volailles), mais limité lorsque le facteur travail est élevé, comme par exemple en production laitière. Dans ces conditions, pour être compétitifs, les petits éleveurs doivent vendre leurs produits à des prix qui ne permettent de rémunérer leur travail qu'à des taux inférieurs à ceux du marché.

Les petits exploitants, aussi bien éleveurs qu'aquaculteurs, affrontent en outre des coûts de transaction supérieurs, en raison des faibles volumes commercialisables et de l'absence d'infrastructure physique et d'infrastructure de marché dans les zones reculées. Les coûts de transaction ont également augmenté là où les producteurs manquent de pouvoir de négociation et d'accès à l'information sur les marchés et demeurent dépendants d'intermédiaires. De plus, l'absence fréquente d'associations de producteurs ou d'autres structures associatives n'aide pas les petits producteurs à réduire leurs coûts de transaction par des économies d'échelle. Enfin, la tendance actuelle à l'établissement de normes et standards visant à la certification des produits de l'élevage et de l'aquaculture sur les marchés occidentaux, risque de contribuer un peu plus à la marginalisation des petits producteurs (Costales et al 2006, FAO 2009b). Bien que le secteur de l'aquaculture soit dominé par les petits producteurs, l'activité est généralement menée comme une entreprise «commerciale», guidée par la maximisation de profits, qu'elle soit de petite comme de grande taille et gérée familialement ou pas (Cai et al 2010). Cela signifie que le temps de l'aquaculture de pure subsistance, où la culture de poissons ou d'autres espèces satisfaisait principalement l'autoconsommation, semble bel et bien révolu.

Concernant la mise en œuvre de politiques pour la gestion de la transition du secteur, trois catégories de petits producteurs doivent être pris en compte, que ce soit dans le cadre de l'élevage comme de l'aquaculture : les petits opérateurs commerciaux qui sont compétitifs et qui peuvent le rester avec des politiques, un soutien institutionnel et des investissements appropriés; les petits producteurs qui pratiquent un élevage domestique uniquement à cause de l'absence d'opportunités alternatives; et les ménages très pauvres qui produisent principalement comme une sorte d'assurance ou de filet de sécurité. Ces catégories requièrent des mesures d'intervention distinctes et spécifiques (World Bank 2007).

\section{2 / Risques pour la santé humaine}

a) Sous et surconsommation de produits d'origine animale

Les produits de l'élevage et de l'aquaculture, tels que la viande, le poisson, les œufs et le lait sont une source très importante de protéines de haute qualité et de micronutriments, notamment dans les pays les moins avancés. Là, le régime alimentaire est essentiellement composé de céréales et tubercules et des déficiences nutritionnelles importantes, spécialement chez les femmes et les enfants, sont communes. Avoir quelques animaux ou un étang poissonnier peut fortement améliorer la qualité du régime si les produits de l'élevage sont consommés régulièrement : la viande rouge est une source importante de fer à haute biodisponibilité, essentiel au développement cognitif des enfants; le lait contribue à une meilleure croissance chez l'enfant (apport de calcium); le foie et les œufs sont une source importante de zinc qui favorise la prévention de la cécité et renforce le système immunitaire; le poisson est une très bonne source d'acides gras de type Omega-3 et Omega-6 qui jouent un rôle clé dans le développement fœtal.

Dans de nombreux pays, en revanche, on observe une réduction des activités physiques combinée à une augmentation de la prise alimentaire, et notamment d'aliments à forte teneur en sucres et en matières grasses et des produits d'origine animale au sein des menus. Le surpoids et l'obésité qui en résultent sont en augmentation rapide dans toutes les régions du globe et affectent tant les adultes que les enfants. Globalement on compte plus d'un milliard de personnes en surpoids (plus que de personnes souffrant d'insécurité alimentaire), dont au moins 300 millions catégorisées comme obèses. Dans les pays en développement à revenu moyen supérieur, l'obésité tend à être associée aux basses classes sociales alors que dans les pays à bas revenu, ce sont plutôt les populations les plus aisées et urbaines qui souffrent d'obésité. 


\section{b) Zoonoses}

Sur vingt cinq maladies affectant l'Homme et considérées comme les plus importantes pour les taux de mortalité ou morbidité qu'elles engendrent, quinze sont originaires des zones tempérées et dix des zones à climat tropical. Les informations actuellement disponibles suggèrent que 8 maladies des quinze originaires des zones tempérées viennent probablement des animaux domestiques (diphtérie, Influenza A, rougeole, oreillons, coqueluche, infections à rotavirus, variole, tuberculose) alors que seules trois maladies (trypanosomoses humaines africaines à Trypanosoma brucei gambiense et à Trypanosoma brucei rhodesiense, maladie de Chagas) des 10 maladies originaires des zones tropicales viendraient des animaux domestiques (Wolfe et al 2007).

$60 \%$ des maladies infectieuses émergentes chez l'Homme décrites entre 1940 et 2004 sont des zoonoses (Jones et al 2008). Le flux important et la forte concentration d'animaux en milieu confiné augmente l'intensité d'exposition aux microbes pour les éleveurs, leurs familles, les employés vétérinaires et autres. Les travailleurs en contact direct avec les animaux sont les plus à risque, surtout dans les nombreux pays ou les réglementations des conditions de travail manquent ou sont mal appliquées. Les systèmes de ventilation, le transport d'animaux et la gestion des effluents d'élevage et des animaux morts constituent des points critiques pour la gestion des risques de transmission.

Les zoonoses aquatiques sont par contre très rares. Les facteurs de transmission de pathogènes sont plus souvent indirects et liés à l'environnement de culture (rétention d'eau en étang: paludisme (moustiques), maladie du sommeil (mouches), dermatite cercarienne (mollusques), cécité des rivières (nématodes)) et/ou dus à une contamination externe lors de la production ou de la transformation des produits aquatiques (contaminants, toxines) se transmettant à l'Homme lors de leur consommation (Turnbull 2010).

Pour faire face aux challenges posés par les problèmes liés aux maladies existantes, à l'augmentation importante du risque de pandémies et de l'émergence de maladies infectieuses en général, la FAO, l'Organisation mondiale de la Santé et l'Organisation Mondiale de la Santé Animale développent une approche modulaire baptisée «Une santé» qui se penche sur les interfaces Hommeanimal-environnement. Cette approche implique un travail multidisciplinaire avec l'intégration de différentes composantes le long des filières. Une approche filière permet en effet d'identifier les points à risque non seulement chez les producteurs mais aussi tout au long de la chaîne de commercialisation. Une représentation graphique des filières crée un cadre dans lequel une expertise interdisciplinaire peut être intégrée afin de planifier et examiner les implications des stratégies de lutte contre les maladies infectieuses telles que la grippe aviaire (McLeod et al 2009). Cette approche est aussi de plus en plus préconisée et utilisée dans le cadre de l'aquaculture.

\section{c) Résistance antimicrobienne}

Un nombre croissant de maladies affectant l'Homme approche le point où elles ne pourront plus être traitées avec les médicaments actuellement disponibles sur le marché. L'augmentation de la résistance aux médicaments, qu'ils soient antiviraux, antiparasitaires ou antibactériens, est le résultat d'une utilisation accrue de ces médicaments en médecine humaine et dans l'élevage, et plus particulièrement l'élevage intensif. La distribution d'antibiotiques mélangés à l'aliment ou à l'eau de boisson est un mode d'administration efficace, notamment en aviculture. Ces modes d'administration représentent néanmoins un risque important d'exposition des bactéries à des doses sublétales, favorisant ainsi la sélection, la persistance et la dissémination de bactéries résistantes aux antibiotiques (Steinfeld et al 2010). Ce problème est important dans tous les pays. Il revêt une importance particulière dans les pays en développement où les différentes espèces se côtoient plus régulièrement, où le matériel nécessaire à un dosage efficace peut faire défaut et où les réglementations et contrôles sont parfois moins stricts par manque de ressources (Steinfeld et al 2010).

Le risque de résistance antimicrobienne est également présent en aquaculture et constitue un danger pour la santé publique, particulièrement là où l'utilisation d'agents antimicrobiens est mal régulée et peu contrôlée, contribuant ainsi à la sélection de bactéries et de gènes résistants et augmentant la présence de résidus antimicrobiens dans les produits aquacoles (FAO/OIE/WHO 2006).

Plusieurs organisations internationales ont développé des codes de conduite et des recommandations pour promouvoir une utilisation responsable et prudente des agents antimicrobiens et contenir le problème de résistance antimicrobienne (par exemple : Organisation Mondiale de la Santé, Organisation Mondiale de la Santé Animale, Codex Alimentarius, FAO). Leur application est cependant incomplète. Il est à noter par ailleurs que, dans le cas de l'aquaculture, la menace ou l'imposition d'interdiction d'importation de produits aquacoles pour raisons sanitaires après découverte d'agents interdits lors de contrôles sanitaires, a eu pour effet de forcer les pays exportateurs à réguler l'utilisation d'agents antimicrobiens et antibactériens afin de pouvoir maintenir leurs débouchés sur les marchés étrangers (Patmasirinwat et al 1999, World Bank 2007). Cela a été le cas, par exemple, pour les crevettes en provenance d'Asie du Sud-Est.

\section{3 / Interactions environ- nementales}

a) Pression sur les ressources naturelles : sols et eau

Le secteur de l'élevage utilise aujourd'hui plus de 3,9 milliards d'hectares près de $30 \%$ de la surface terrestre du globe - principalement sous forme de pâturages. On estime que 500 millions d'hectares sont cultivés en plantes fourragères (35\% des terres cultivables), 1,4 milliard d'hectares sont des pâturages à haute productivité et les 2 milliards d'hectares restant sont des pâturages de type extensif à productivité relativement faible (FAO 2006).

Il est intéressant de noter que malgré une forte augmentation quantitative et qualitative en production animale, une forte augmentation des productions animales terrestres, le volume mondial de céréales utilisé pour l'aliment bétail a diminué de $40 \%$ sur les deux dernières décennies. Ceci est dû à une forte amélioration de l'indice de conversion et à un déplacement de la production des ruminants vers les espèces monogastriques qui nécessitent moins de céréales par unité de viande produite. La forte diminution de l'utilisation de céréales fourragères dans les anciennes économies soviétiques après leur transition d'une économie planifiée vers une économie de marché, ainsi que le changement des politiques de subsides de l'Union Européenne ont aussi joué un rôle important dans la diminution de l'utilisation de céréales. Mais ces tendances ne vont pas se poursuivre. Il est prévu une augmentation dans la demande totale en aliment bétail d'environ $1,6 \%$ par année pour atteindre 1,1 milliard de Tonnes en 2030. Cette demande va nécessiter une nouvelle augmentation de la productivité par hectare mais aussi une augmentation de la surface cultivée (FAO 2009b). Il est cependant prévisible que cette augmentation ne se fasse pas sans heurts étant donné la compétition croissante pour les ressources naturelles, notamment foncières et hydriques. 
Les tendances observées dans les systèmes d'élevage extensifs sont plus complexes que celles observées dans les systèmes intensifs. On observe une expansion sur des terres productives caractérisées par de relativement faibles coûts d'opportunité en Amérique Latine, une forte diminution en pâturages fertiles et une limitation de la mobilité en Afrique subsaharienne et en Asie et la récupération de prairies abandonnées dans les économies en transition (Steinfeld et al 2010).

Le secteur de l'élevage joue un rôle déterminant dans l'augmentation de l'utilisation des ressources en eau, responsable de plus de $8 \%$ de l'eau prélevée pour l'utilisation humaine au niveau mondial, principalement pour l'irrigation des cultures fourragères. Les sources principales de pollution sont les déchets d'origine animale, les antibiotiques et les hormones, les produits chimiques des tanneries, les engrais et pesticides utilisés pour les cultures fourragères et les sédiments des pâturages érodés. L'élevage affecte également la reconstitution des nappes phréatiques en compactant les sols, en réduisant l'infiltration et en détériorant les rives des cours d'eau.

On peut réduire l'utilisation de l'eau en améliorant l'efficacité des systèmes d'irrigation. Il est possible de faire face à l'impact de l'élevage sur l'érosion, la sédimentation et la régulation des eaux en prenant des mesures pour lutter contre la dégradation des terres. On peut également limiter la pollution grâce à une meilleure gestion des déchets d'origine animale au sein des unités de production industrielle, à une meilleure alimentation facilitant l'absorption des nutriments, à une meilleure gestion du fumier (notamment par la méthanisation) et à une meilleure utilisation des déjections transformées sur les terres agricoles. La production animale industrielle devrait notamment être redirigée vers les terres agricoles accessibles où les déchets peuvent être recyclés sans surcharger les sols.

L'aquaculture est également source de pollution lorsque certains systèmes de culture trop intensifs conduisent à un dépassement de la capacité de charge des environnements dont ils dépendent (cas de l'impact des rejets polluants de la crevetticulture sur la croissance des mangroves en Thaïlande, Vaiphasa et al 2007 ; cas du développement de la culture en cage de tilapia dans des réservoirs hydroliques en Indonésie, Abery et al 2005). En réponse, l'aquaculture multitrophique, qui intègre dans le même système de production des espèces issues de niveaux trophiques et/ou nutritionnels différents, est en expan- sion. Cette pratique favorise la durabilité économique et environnementale. Cependant, les déchets d'une espèce devenant l'apport nutritionnel pour d'autres, des problèmes de sécurité sanitaire et de qualité des aliments se posent. Cette pratique étant nouvelle, des recherches devront être effectuées dans ce domaine afin de s'assurer que le poisson ainsi produit ne présente pas de risque pour les consommateurs (Soto 2009).

D'autres formes d'intégration existent. L'aquaculture intégrée à l'agriculture, comme par exemple la rizipisciculture, très pratiquée en Asie, est un moyen reconnu pour optimiser l'utilisation des ressources foncières et aquatiques et d'améliorer les moyens d'existence des foyers (Asie : FAO/ ICLARM/IIRR 2001, Hong 2007 ; Afrique : Dey et al 2010). L'aquaculture intégrée à l'irrigation, afin d'améliorer la productivité de l'eau (i.e. quantité de nourriture produite par $\mathrm{m}^{3}$ d'eau utilisé) est rencontrée dans les petits périmètres irrigués (Halwart et van Dam 2006). Bien que préconisée aussi dans les grands périmètres irrigués (Haylor et Bhutta 1997), elle y demeure marginale pour des raisons techniques ( $\mathrm{Li}$ et al 2005) et institutionnelles (Brugère 2006). Enfin, l'aquaponie, un système clos et intégré de culture de végétaux en symbiose avec un élevage de poissons fait l'objet d'une attention croissante. Grâce au recyclage des nutriments, une réduction des décharges dans l'environnement et une meilleure utilisation de l'eau, l'aquaponie peut permettre de faire face aux contraintes de disponibilités en terres et en eau, et peut ainsi être développée en climat aride, mais les coûts des infrastructures nécessaires à ce type de production restent élevés (Rakocy et al 2006).

\section{b) Changement climatique}

Avec l'augmentation des températures, la montée du niveau des mers, les modifications des courants maritimes et des schémas météorologiques, le changement climatique est le plus grand défi environnemental que doit affronter l'espèce humaine. Le secteur de l'élevage joue un rôle majeur dans les émissions de gaz à effet de serre, étant responsable de $18 \%$ des émissions, si on suit une analyse en cycle de vie (FAO 2006).

L'élevage est à l'origine de $9 \%$ des émissions anthropiques de dioxyde de carbone. L'essentiel résulte des changements d'utilisation des terres - surtout la déforestation - provoqués par l'expansion des pâturages et des terres arables destinées aux cultures fourragères. Le secteur émet $37 \%$ du méthane anthropique (dont le potentiel de réchauffement global est 23 fois plus élevé que celui du $\mathrm{CO}_{2}$ ), issu pour la plupart de la fermentation entérique des ruminants, et $65 \%$ de l'hémioxyde d'azote, découlant principalement du fumier.

Le pendant à ce niveau élevé d'émissions est le grand potentiel d'atténuation des émissions dans le secteur de l'élevage. L'amélioration de la productivité (à la fois en production animale et en culture fourragère), peut réduire l'intensité des émissions de gaz à effet de serre (FAO 2006). De plus, reconstituer les stocks de carbone dans le sol grâce au labour de conservation, aux cultures de couverture, à l'agroforesterie et à d'autres mesures, pourrait permettre de piéger jusqu'à 1,3 Tonne de carbone par hectare et par an, sans compter les quantités obtenues par la restauration des pâturages désertifiés. Les émissions de méthane peuvent être réduites grâce à une meilleure alimentation, réduisant la fermentation entérique, à une meilleure gestion du fumier ainsi qu'aux biogaz, qui fournissent également une énergie renouvelable. Il est possible de diminuer les émissions d'azote en améliorant l'alimentation et la gestion du fumier.

Etant donné qu'environ $80 \%$ de la production aquacole mondiale est basée sur la culture d'herbivores ou d'omnivores d'eau douce nécessitant peu de fertilisants et de provende, l'aquaculture génère, de manière générale, moins d'émissions de gaz à effet de serre - à l'exception de la crevetticulture et de la salmoniculture - que l'élevage (Gerber et al 2007, Tyedmers et Pelletier 2007). Bien que les systèmes aquatiques de production (casiers à riz, bas-fonds, étangs sédimentaires) émettent du méthane (en quantité cependant encore non quantifiée) les organismes aquatiques cultivés ne génèrent pas d'émissions, réduisant ainsi la quantité totale de gaz à effet de serre émise par unité de poisson (ou dérivés) produite.

Il est à noter que l'élevage comme l'aquaculture contribuent également à l'adaptation au changement climatique. Le type d'adaptations possibles varie selon la nature des activités pratiquées (à petite ou grande échelle, commerciales ou plutôt de subsistance), les systèmes de productions et les espèces cultivées. Ainsi, les productions animales intégrées à l'agriculture permettent de recycler les nutriments et d'utiliser les ressources naturelles plus efficacement lorsque celles-ci sont menacées par les changements climatiques (Soto et al 2008). Des cycles de production courts, un choix judicieux d'espèces et de nouvelles technologies ou de gestion 
permettent aussi de saisir des opportunités saisonnières. L'aquaculture offre aussi un moyen d'adaptation à d'autres secteurs affectés négativement par les effets des changements climatiques. Par exemple, dans les régions deltaïques d'Asie, où l'agriculture et l'aquaculture continentale sont dominantes et menacées par la salinisation et la montée du niveau de la mer, la culture d'espèces euryhalines (tolérant la salinité) dans des étangs creusés là où il n'est plus possible de cultiver des céréales, peut jouer un rôle majeur dans le maintien des moyens d'existence locaux (De Silva et Soto 2009).

\section{c) Gestion de la biodiversité}

L'élevage a une forte influence sur la biodiversité du fait de l'importance des surfaces mobilisées. Dans de nombreux systèmes mixtes et extensifs, on observe une co-évolution entre pratiques de production et biodiversité, l'élevage participant ainsi à la genèse et au maintien de la diversité. Les évolutions rapides des techniques de production (gestion des effluents, des prairies, des cultures pour l'aliment) ont toutefois généralement des implications négatives sur la biodiversité. Ces impacts sont principalement liés à l'incapacité des espèces naturelles à s'adapter suffisamment rapidement aux transformations des habitats et à l'altération des ressources (climat, qualité de l'air et de l'eau, dégradation des terres et déforestation). De plus, certains conflits relatifs aux ressources entre éleveurs et espèces de prédateurs sauvages menacent non seulement ces dernières mais aussi les zones protégées proches de pâturages.

L'intensification des systèmes d'élevage peut aussi réduire l'appropriation des habitats de la faune sauvage par l'élevage (Steinfeld et al 2010). La protection des zones sauvages, des zones tampons et des servitudes de conservation peuvent augmenter la superficie des terres où la conservation de la biodiversité est prioritaire. Des efforts accrus devraient être déployés en faveur de l'intégration de la production animale et des éleveurs dans la gestion des paysages.
Malgré des impacts pouvant être positifs (par exemple à travers une réduction de la surexploitation des stocks d'espèces sauvages), le développement de l'aquaculture pèse aussi sur la biodiversité des écosystèmes sur lesquels elle repose, à cause de ses rejets de polluants et de modification des habitats sensibles et des niches écologiques (Diana 2009). Cependant, et contrairement à l'élevage, c'est l'introduction d'espèces exotiques domestiquées, qui menace le plus la biodiversité locale, soit au travers de leur relâche dans l'environnement lors d'erreurs de manipulation, ou lors de la transmission de maladies aux espèces indigènes sauvages.

L'élevage et l'aquaculture jouent un rôle important dans la surexploitation des stocks halieutiques, du fait de leur demande en farines et huiles de poisson destinées à la fabrication de provende. La surexploitation des pêches a affecté la taille et la viabilité des populations de poissons, la génétique des espèces cibles, ainsi que les chaînes alimentaires et les écosystèmes dont elles font partie (Bell et Waagbo 2008). Environ $17 \%$ de la farine de poisson produite dans le monde est fabriquée à partir des déchets issus de la transformation de poisson de consommation avec un impact limité sur les stocks de poisson. Cependant, les $83 \%$ restants proviennent de la pêche de capture marine directe (FAO 2006). Les farines de poisson sont utilisées à part relativement égale entre l'aquaculture et l'élevage, mais la part de l'aquaculture croît du fait de la plus forte croissance de cette dernière (Rana et al 2009).

L'exténuation des stocks incite les secteurs de l'aquaculture à réduire la part des farines et huiles de poissons dans l'aliment (tableau 4). Les recherches en nutrition, visant à réduire la dépendance du secteur avicole et piscicole vis-à-vis de la farine de poisson posent toutefois des questions d'ordre éthique liées au fait de nourrir des poissons à base de céréales et résidus animaux et de nourrir des espèces carnivores destinées à des marchés de luxe avec des farines à base de poissons qui pourraient autrement être destinés à la consommation humaine, en particulier dans les pays où les protéines animales font défaut (Tacon et al 2006, Hasan et Halwart 2009).

\section{4 / Synthèse}

L'analyse comparée de l'élevage et de l'aquaculture fait émerger des similitudes et différences entre ces deux formes de production animale, ainsi que des enjeux communs aux deux sous-secteurs.

Les productions de volailles (viande et œufs) et de poissons omnivores sont en très forte croissance, répondant de manière particulièrement efficace à une demande accrue en produits animaux. Elles sont basées sur des espèces à court cycle et des aliments à forte valeur nutritive, et leurs produits sont souvent substituables sur les marchés et dans les préparations alimentaires. Se modernisant rapidement, elles devancent les règlementations et opèrent souvent dans des contextes politiques insuffisants (FAO 2009a et b). Elles font en outre face à des défis similaires, en matière d'environnement, de résistance microbienne et de contribution à la sécurité alimentaire, utilisant des ressources céréalières et halieutiques qui pourraient autrement être utilisées dans 1'alimentation des populations humaines. $\mathrm{La}$ production porcine se caractérise par une croissance moindre et une plus faible efficacité de conversion de l'aliment (FAO 2009b). En outre, la substitution entre viande porcine et produits de l'aquaculture est limitée.

Du point de vue du taux de conversion alimentaire, la production de poissons carnivores peut quant à elle se comparer aux formes intensifiées de production de ruminants. Ces espèces sont en effet moins efficaces quant à la conversion d'aliments et requièrent des volumes (ruminants) ou des qualités (poissons carnivores) d'aliments supérieurs par unité de produit. Il en résulte de plus forts impacts sur les ressources

Tableau 4. Objectifs de réduction de l'utilisation de farines et huiles de poisson dans la préparation d'aliment pour l'aquaculture.

\begin{tabular}{lcccc}
\hline Espèce & \multicolumn{2}{c}{$\begin{array}{c}\text { Utilisation actuelle } \\
(\%)\end{array}$} & \multicolumn{2}{c}{$\begin{array}{c}\text { Utilisation estimée à moyen/long terme } \\
(\%)\end{array}$} \\
\hline & farines de poisson & huiles de poisson & farines de poisson & huiles de poisson \\
\hline Saumon de l'atlantique & $35-47$ & $25-33$ & $12-16$ & $8-12$ \\
Truite arc-en-ciel & $30-35$ & $20-25$ & 5 & 5 \\
Carpe commune & $5-25$ & $5-10$ & 0 & 0 \\
\hline
\end{tabular}

Source : Rana et al 2009. 
naturelles ainsi que des coûts de production plus élevés. Ces produits répondent à la demande d'une population relativement aisée, qui augmente principalement dans les pays émergents. Concernant les ruminants, les systèmes d'élevage extensif, notamment dans les pays les moins développés, contribuent au contraire fortement à la sécurité alimentaire des populations rurales et ne rentrent pas dans cette catégorie.

L'élevage intensif comme l'aquaculture est dépendant des prix de l'aliment, qui compte pour 60 à $80 \%$ des coûts de production. Lorsque, entre 2005 et 2008 , les cours du mais, du riz et du soja ont approximativement triplé, l'effet à été immédiat sur les coûts de la production animale et donc les prix des produits de l'élevage et de l'aquaculture. Pour la première fois depuis des dizaines d'années, les prix réels du poisson ont augmenté (FAO 2009a). L'aquaculture est donc particulièrement sujette à la volatilité des marchés internationaux. Ce phénomène est inquiétant pour le développement futur du secteur, mettant en péril non seulement la pérennité des petits producteurs mais plus généralement le rôle du secteur comme moteur de développement rural et comme fournisseur de protéines alimentaires (Rana et al 2009). Il est à noter que, au cours de la même période, les prix des espèces issues des pêches de capture augmentèrent plus que ceux des espèces cultivées en raison de l'impact plus fort de la hausse des prix de l'énergie sur les opérations des navires de pêche.

La rapide transformation des systèmes de productions animales s'est opérée loin des yeux du consommateur. Il en résulte une méconnaissance des modes de production et de transformation et des crises de confiance alimentées par des incidents sanitaires (ESB, fièvre aphteuse, Influenza H1N1, contamination à la dioxine ou à la mélanine) et des préoccupations éthiques (bienêtre animal). Si le phénomène concerne plus particulièrement l'élevage, il touche également l'aquaculture. L'aquaculture et l'élevage biologiques sont des formes de réaction à ces problèmes, qui attirent l'attention de certains consommateurs, des défenseurs de l'environnement et d'entrepreneurs novateurs. Ils peuvent réduire l'exposition aux substances toxiques émanant des pesticides qui peuvent s'accumuler dans le sol, l'air, l'eau et les approvisionnements alimentaires, diminuant ainsi les risques pour la santé des consommateurs. Ces formes de production présentent également d'autres avantages, notamment une meilleure gestion de la fertilité des sols et des intrants, la protection les eaux souterraines et facilitent les écono- mies d'énergie. Par ailleurs, les normes biologiques interdisent l'utilisation du génie génétique dans la production, ce qui rassure aussi les consommateurs. L'intérêt croissant que suscite la production biologique a poussé les gouvernements à réglementer le secteur. Des normes et des procédures de certification sont actuellement en cours d'élaboration dans le domaine de l'aquaculture ; il s'agit d'instruments nécessaires pour promouvoir l'investissement, garantir la provenance, l'éthique de production et la qualité des produits auprès des consommateurs. En l'absence de normes internationales, les parties intéressées élaborent leurs propres normes et organes d'accréditation. Ces normes varient souvent de manière importante selon le lieu, le certificateur et l'espèce, et leurs coûts de mise en œuvre peuvent exclure de facto les petits producteurs des opportunités offertes par le commerce international de leurs produits.

La contribution de l'aquaculture et de l'élevage à la croissance économique est indéniable : création d'emplois directs et indirects en amont et aval de la chaîne, salaires, génération de revenus directs pour les exploitants et indirects pour les gouvernements à travers le commerce international et l'imposition de taxes. Smith et al (2010) démontrent les bénéfices économiques et nutritionnels que peut générer le commerce de produits aquatiques pour les foyers pauvres. Ils soulignent cependant la précarité de ces bénéfices si la production ne s'opère pas dans le cadre de régulations et institutions visant à la gestion durable des ressources naturelles, à la clarification de l'accès au foncier et à la redistribution équitable des revenus. De même, les objectifs de réduction de la pauvreté, de sécurité alimentaire, de développement humain et d'amélioration du statut des femmes ne sont entièrement atteints que grâce à l'intervention des pouvoirs publics pour protéger les intérêts des petits producteurs (Cai et al 2010). C'est par exemple le cas en Asie avec l'interdiction de transfert des permis ou des licences, des limitations de taille de fermes et un devoir de coopération entre larges et petits producteurs (Hishamunda et al 2009). De plus, il est montré qu'un accès limité au foncier peut conduire à une mauvaise gestion et à une production réduite des activités aquacoles, même lorsque les prix de marché sont en augmentation (Smith et al 2010).

Si l'élevage de volailles et porcins s'est largement développé en l'absence de subventions, le développement de l'aquaculture et de certaines formes d'élevage de ruminants (notamment dans l'Union Européenne) a été marqué par des politiques d'interventions direc- tes. Il est nécessaire de trouver un équilibre entre intervention étatique et libre entreprise dans la formulation des politiques de développement du secteur (Brugère et al 2010). Avec d'un côté la flambée des prix et d'un autre le maintien de niveaux de pauvreté intolérables dans de nombreuses parties du globe, il est par ailleurs envisagé que les gouvernements et les organisations internationales devront encourager la production de denrées alimentaires (dont celles issues de l'élevage et de l'aquaculture) au-delà de celle purement guidée par les mécanismes de marché afin de stabiliser les prix et de garantir l'équité de l'accès aux opportunités de production (Charles et al 2010).

\section{Conclusions}

Les productions animales sont engagées dans un processus de croissance et de transformation depuis une cinquantaine d'années, bien que la tendance se soit accélérée plus récemment pour l'aquaculture. Cette tendance va se maintenir, principalement alimentée par la croissance du pouvoir d'achat des populations urbaines.

La compétition accrue pour les ressources naturelles et la croissance de la demande globale de nourriture forcent les deux sous-secteurs à s'adapter et produire mieux avec moins, ouvrant la voie au développement de technologies nouvelles, notamment concernant les indices de conversion et au développement d'institutions plus performantes.

Les productions animales, grâce à leur forte croissance et leur rapide transformation ont souvent devancé le développement de politiques publiques. $\mathrm{Ce}$ processus s'est parfois traduit par une mauvaise gestion des biens publics et requiert le développement de politiques et institutions adaptées. Ce faisant, les décideurs politiques doivent prendre en compte les multiples fonctions que remplissent les productions animales dans les moyens d'existence et la sécurité alimentaire des populations les plus pauvres. La nécessité d'intervention de l'Etat dans le développement des productions animales est reconnue et doit se concentrer sur un rôle de cadrage. Les interventions devraient avoir pour objectif d'établir un contexte institutionnel (gouvernance) stable et adéquat et un cadre régulateur minimal de façon à éviter les conflits avec les autres secteurs ainsi que les impacts néfastes sur l'environnement, les communautés et sur l'industrie elle-même.

Trois domaines se distinguent, ayant chacun leurs priorités : 
- Concernant la sécurité sanitaire : l'analyse et le renforcement de la résilience des systèmes vis-à-vis des zoonoses, ainsi que le contrôle des réservoirs de multirésistance aux médicaments qui existent déjà chez l'Homme et chez l'animal. Seule une réponse globale à l'actuelle recrudescence des résistances aux antibiotiques, intégrant l'utilisation d'antimicrobiens dans les différents systèmes de production animale et chez l'Homme pourra être efficace.
- Concernant les ressources naturel les : l'amélioration de l'efficience des systèmes, conçue comme la quantité de ressources naturelles mobilisées ou de pollutions émises par unité de produit, ainsi que la compréhension et la prise en compte de la valeur des services écosystémiques dans les coûts de production permettront de ralentir la dégradation des ressources naturelles sur lesquelles reposent les productions animales.
- Concernant la réduction de la pauvreté et le développement rural: le maintien de conditions de concurrence équitables pour les petits producteurs, le soutien à l'innovation technologique et institutionnelle et la mise en place de systèmes de protection sociale seront nécessaires pour garantir à l'élevage et à l'aquaculture leur rôle à jouer dans l'atteinte des objectifs de développement du millénaire.

\section{Références}

Abery N.W., Sukadi F., Budhiman A.A., Kartamihardja E.S., Koeshendrajana S., Buddhiman De Silva S.S., 2005. Fisheries and cage culture of three reservoirs in west Java, Indonesia; a case study of ambitious development and resulting interactions. Fish. Manag. Ecol., 12, 315-330.

ASC-WWF, 2010. Pangasius Aquaculture Dialogue Standards/Bivalve Aquaculture Dialogue Standards. Aquaculture Stewardship Council and World Wildlife Fund for Nature, August 2010.

Bell J.G., Waagbo R, 2008. Safe and nutritious aquaculture produce: benefits and risks of alternative sustainable aquafeeds. In: Aquaculture in the Ecosystem. Holmer M. Black K., Duarte C.M., Marba N., Karakassis I. (Eds). Springer, Berlin, Allemagne, 185-225.

Bostock J., McAndrew B., Richards R. Jauncey K., Telfer T., Lorenzen K., Little D., Ross L., Handisyde N. Gatward R., Corner R., 2010. Aquaculture: Global status and trends. Philos. Trans. Royal Soc. Biol. Sci., 365, $2897-$ 2912. doi: 10.1098/rstb.2010.0170

Brugère C., 2006. A review of the development of integrated irrigation aquaculture (IIA), with special reference to West Africa. In: Integrated irrigation and aquaculture in West Africa: Concepts, practices and potential. Halwart M., van Dam A.A. (Eds). FAO, Rome, 27-59.

Brugère C., Ridler N., Haylor G., Macfadyen G., Hishamunda N., 2010. Aquaculture planning: policy formulation and implementation for sustainable development. FAO Fish. Aquacult. Technical Paper, 542, 70p.

Cai J., Jolly C., Hishamunda N., Leung P.S., 2010. Review on aquaculture and socio-economic growth and development: enabling policies, legal framework and partnership for improved benefits. Global Conference on Aquaculture, 22-25 September 2010, Phuket, Thailand. NACA/DoF/FAO.

Charles H., Godfry J, Beddington J.R. Crute I.R., Haddad L., Lawrence D., Muir J.F., Pretty J., Robinson S., Thomas S.M., Toulmin C., 2010. Food security: The challenge of feeding 9 billion people. Science, 327, 812-818

Costales A., Gerber P. Steinfeld H., 2006. Underneath the livestock revolution. In: The 2006 Livestock Report. McLeod A. (Ed). FAO, Rome, Italie, 102p.

De Silva S.S., Soto D., 2009. Climate change and aquaculture: potential impacts, adaptation and mitigation. In: Climate change implications for fisheries and aquaculture. Overview of current scientific knowledge. Cochrane K., Young C. de, Soto D., Bahri T.
(Eds). FAO Fish. Aquacult. Technical Paper, $530,139-216$

Dey M.M., Garcia Y. T., Kumar P., Piumsombun S., Haque M. S., Li L., Radam A., Senaratne A., Khiem N. T., Koeshendrajana S., 2008. Demand for fish in Asia: A CrossCountry Analysis. Aust. J. Agric. Res. Econ., $52,321-338$.

Dey M.M., Paraguas F.J., Kambewa P., Pems D.E., 2010. The impact of integrated aquaculture-agriculture on small-scale farms in Southern Malawi. Agricult. Econ., 41, 1, 67-79.

Diana J.S., 2009. Aquaculture production and biodiversity conservation. Bioscience, 59 , 1, 27-38.

FAO, 2006. Livestock's long shadow. FAO, Rome, Italie, 26p

FAO, 2007. The State of the World Fisheries and Aquaculture 2006. FAO, Rome, Italie, $180 \mathrm{p}$

FAO, 2008. L'état des ressources zoogénétiques pour l'alimentation et l'agriculture dans le monde, FAO, Rome, Italie, 43p.

FAO, 2009a. The State of World Fisheries and Aquaculture 2008. FAO, Rome, Italie, 196p.

FAO, 2009b. La situation mondiale de l'alimentation et de l'agriculture. Le point sur l'élevage. FAO, Rome, Italie, 196p.

FAO, 2010a. Salmon highlights July 2010 Department of Fisheries and Aquaculture, FAO, Rome, Italie.

FAO, 2010b. Moving aquaculture further offshore: Governance issues and challenges Committee on Fisheries, $5^{\text {th }}$ Session of SubCommittee on Aquaculture, 27 September-01 October 2010, Phuket, Thailand. FAO, Rome, Italie, $13 \mathrm{p}$.

FAO, 2010c. State of World Fisheries and Aquaculture 2010. Food and Agriculture Organization, Rome, Italie, 218p

FAOSTAT, 2010. Food and Agriculture Organization Statistical Database. http://faostat.fao.org/site/339/default.aspx

FAO/ICLARM/IIRR, 2001. Integrated agriculture-aquaculture : A primer. FAO Fish Technical Paper, 407, FAO, Rome, Italie.

FAO/OIE/WHO, 2006. Report of a joint FAO/OIE/WHO expert consultation on antimicrobial use in aquaculture and antimicrobial resistance, 13-16 June 2006, Seoul, Republic of Korea. WHO, Geneva, Suisse.

Forster J., 2002. Farming salmon: An example of aquaculture for the mass market. Rev. Fish. Sci., 10, 577-591.
Gerber P., Mooney H.A., Dijkman J., de Haan C., Tarawali S., 2010. Livestock in a changing landscape: Experiences and regional perspectives. Island Press, Washington D.C., USA, 396p.

Gerber P., Wassenaar T., Sosales M., Castel V., Steinfeld H., 2007. Environmental impacts of a changing livestock production: Overview and discussion for a comparative assessment with other food production sectors. In: Comparative assessment of the environmental costs of aquaculture and other food production sectors: Methods for meaningful comparisons. Bartley D., Brugère C., Soto D., Gerber P., Harvey B. (Eds). FAO Fish. Proc., 10, 34-54.

Globefish, 2009. Pangasius market report, July 2009, FAO GLOBEFISH, http://www .globefish.org/dynamisk.php4?id=4740

Halwart M., van Dam A.A.,2006. Integrated irrigation and aquaculture in West Africa: Concepts, practices and potential. FAO, Rome, Italie, $180 \mathrm{p}$.

Hasan M.R., Halwart M., 2009. Fish as feed inputs for aquaculture: practices, sustainability and implications. FAO Fish. Aquacult. Technical Paper, 518, 426p.

Haylor G., Bhutta M.S., 1997. The role of aquaculture in the sustainable development of irrigated farming systems in Punjab, Pakistan. Aquacult. Res., 28, 691-705.

Hishamunda N., Ridler N., 2002. Macro policies to promote sustainable commercial aquaculture. Aquacult. Int., 10, 491-505.

Hishamunda N., Bueno P.B., Ridler N., Yap W.G., 2009. Analysis of aquaculture development in Southeast Asia: a policy perspective. FAO Fish. Aquacult. Technical Paper, 509, 78p.

Hong S., 2007. Le stockage, la production et la collecte de poisons dans les casiers à riz irrigués dans le bas bassin du Mekong. Synthèse technique. ENGREF, Montpellier. http://www.agroparistech.fr/IMG/pdf/Hong.pdf Consulté le 11.10.2010.

Jones K.E., Patel N.G., Levy M.A., Storeygard A., Balk D., Gittleman J.L., Daszak P., 2008. Global trends in emerging infectious diseases, Nature, doi:10.1038/nature06536, 451, 990-994.

Knapp G., Roheim C.A., Anderson J.L., 2007. The Great Salmon Run. Competition between wild and farmed salmon. Traffic North America. Institute of Social and Economic Research, University of Alaska, Anchorage, 43p.

Lee C.S., 2005. Application of biosecurity in aquaculture production systems. In: Aquaculture and Pathobiology of Crustacean 
and Other Species. Proceedings of the ThirtySecond U.S. Japan Symposium on Aquaculture. Sakai Y., McVey J.P., Jang D., McVey E., Caesar M. (Eds). NOAA Research, Silver Spring, MD, UJNR Technical Report, 32, 66-75. http://www.lib.noaa.gov/retiredsites/japan/aquaculture/proceedings/report32/1 ee corrected.pdf. consulté le 09.09.2010.

Li Q., Gowing J.W., Mayilswami C., 2005. Multiple-use management in a large irrigation system: an assessment of technical constraints to integrating aquaculture within irrigation canals. Irrigation and Drainage, 54, 31-42.

Marine Harvest, 2010. Salmon Farming Industry Handbook. Marine Harvest Plc., 68p.

McLeod A., Kobayashi M., Gilman J. SIagian A., Young M., 2009. The use of poultry value chain mapping in developing HPAI control programs. World's Poult. Sci. J., 65, 217-223.

Naylor R.L., Hardy R.W., Bureau D.P,, Chiu A., Elliot M., Farrell A.P., Forster I., Gatlin D.M., Goldburg R.J., Hua K., Nichols P.D., 2009. Feeding aquaculture in an era of finite resources. Proc. Nat. Acad. Sci. USA, 106, 36, 15103-15110.

Nurdjana M.L., 1999. Development of shrimp culture in Indonesia. Paper presented at the Bangkok FAO technical consultation on policies for sustainable shrimp culture, Bangkok, Thailand, 8-11 December 1997. Rome, Italie. FAO Fisheries Report, 572.

Patmasirinwat P., Bennis M., Pednekar S., 1999. International trade, environmental issues and the impact on sustainability of shrimp culture in Thailand. In: Towards sustainable shrimp culture in Thailand and the region. Smith P. (Ed) Australian Centre for International Agricultural Research, Canberra, Australie, 132-141.
Pike I.H., 2005. Eco-efficiency in aquaculture: global catch of wild fish used in aquaculture. Int.Aquafeed, 8, 38-40.

Rakocy J.E., Masser M.P., Losordo T.M 2006. Recirculating aquaculture tank production system: Aquaponics. Integrating fish and plant culture. Southern Regional Aquaculture Center. Mississippi State University, Stoneville, Mississippi, USA. Publ. 454, 5p.

Rana K.J., Siriwardena S., Hasan M.R. 2009. Impact of rising feed ingredient prices on aquafeeds and aquaculture production. FAO Fish. Aquacult. Technical Paper, 541, 63p.

Rubino M., 2008. Offshore Aquaculture in the United States: Economic considerations, implications \& opportunities. NOAA Technical Memorandum NMFS F/SPO-103. U.S Department of Commerce; Silver Spring, MD, USA. Rich K.M., 2009. What can Africa contribute to global meat demand? Opportunities and constraints. Outlook on Agric., 38, 223-233.

Smith M.D., Roheim C.A., Crowder L.B. Halpern B.S., Turnipseed M., Anderson J.L., Asche F., Bourillón L., Guttormsen A.G.A., Khan L.A. Liguori McNevin A., O'Conno M.I., Squires D., Tyedmers P., Brownstein C. Carden K., Klinger D.H., Sagarin R., Selkoe K.A., 2010. Sustainability and global seafood. Science, 327, 784-786.

Soto D., 2009. Integrated mariculture: A global review. FAO Fish. Aquacult. Technical Paper, 529, 183p.

Soto D., Aguilar-Manjarrez J., Hishamund N., 2008. Building an ecosystem approach to aquaculture. FAO, Rome, Italie, FAO Fisheries and Aquaculture Proc., 14, 221p.

Steinfeld H., Mooney H., Schneider F., Neville L., 2010. Livestock in a changing landscape: drivers, consequences, and responses. Island Press, Washington D.C., USA, 416p.
Tacon A.G.J., Hasan M. R., Subasinghe R.P., 2006. Use of fishery resources as feed inputs for aquaculture development: Trends and policy implications. FAO, Rome, Italie, FAO Fisheries Circular, 1018, 21p.

Turnbull J., 2010. Aquaculture and human health. aquatic veterinary studies and aquatic pathobiology course presentation. Institute of Aquaculture, University of Stirling, UK http://www.staff.stir.ac.uk/j.f.turnbull/12 06LivZoonosis.pdf. consulté le 09.09.2010

Tyedmers P.H., Pelletier N., 2007. Biophysical accounting in aquaculture: insights from current practices and the need for methodological development. In: Comparative assessment of the environmental costs of aquaculture and other food production sectors: Methods for meaningful comparisons. Bartley D.M., Brugère C., Soto D., Gerber P., Harvey B. (Eds). FAO/WFT Expert Workshop, 24-28 April 2006, Vancouver, Canada. FAO Fisheries Proc., 10, 229-241.

Vaiphasa C., de Boer W.F., Skidmore A.K., Panitchart S., Vaiphasa T., Bamrongrugsa N., Santitamnont P., 2007. Impact of solid shrimp pond waste materials on mangrove growth and mortality: a case study from Pak Phanang, Thailand. Hydrobiologia, 591, 47-57.

Wolfe N.D., Panosian Dunavan C., Diamond J., 2007. Origins of major human infectious diseases. Nature, doi:10.1038/nature05775, 447, 279-283.

World Bank, 2007. Changing the face of the waters. Meeting the Promise and Challenge of Sustainable Aquaculture. The World Bank, Washington D.C., USA, 210p.

World Bank, 2010. World development indicators. The World Bank, Washington D.C., $498 \mathrm{p}$.

\section{Résumé}

Les productions animales sont engagées dans un rapide processus de croissance et de transformation. Cette tendance devrait se maintenir dans les prochaines décennies, principalement alimentée par la croissance du pouvoir d'achat des populations urbaines. L'analyse comparée de l'élevage et de l'aquaculture fait émerger des similitudes et des différences entre ces deux formes de production animale. Les productions de volailles et de poissons omnivores sont en très forte croissance, répondant de manière particulièrement efficace à la demande. Elles sont basées sur des espèces ayant un cycle reproductif court et des aliments à haute valeur nutritive, et leurs produits sont souvent substituables. Du point de vue du taux de conversion alimentaire, la production de poissons carnivores peut quant à elle être comparée aux formes intensifiées de production de ruminants. Ces espèces sont en effet moins efficaces quant à la conversion d'aliments et requièrent des volumes (ruminants) ou des qualités (poissons carnivores) d'aliments supérieurs par unité de produit. La particularité des espèces ruminantes est toutefois de pouvoir se nourrir entièrement de ressources végétales sans valeur nutritive pour l'Homme. La croissance de la demande globale et la compétition accrue pour les ressources naturelles forcent les deux sous-secteurs à s'adapter et à produire mieux avec moins. Les productions animales, de par leur forte croissance et leur rapide transformation ont souvent devancé le développement de politiques publiques. Ce processus s'est parfois traduit par une mauvaise gestion des biens publics et requiert le développement de politiques et institutions adaptées.

\section{Abstract}

Changes in global livestock and aquaculture sectors: a review of trends and their implications for societies and the environment

Animal production undergoes rapid growth and structural changes. This trend will continue over the coming decades, driven primarily by an increasing purchasing power among urban populations. A comparative analysis of livestock production, including terrestrial animals, and aquaculture, brings out both similarities and differences between these two sectors. Both poultry and omnivorous fish production are growing rapidly, effectively meeting demand for animal proteins. This sustained growth is possible thanks to the use of short cycle species with high feed conversion ratios. Products from these two sub-sectors also compete on similar markets. On the other hand, carnivorous fish production may are comparable to intensive ruminant production for their greater feed require- 
ments, either quantity or quality wise. Ruminants can however also feed entirely on plant material that has no use for human consumption and thus broaden the trophic base of societies. The increase in global demand for animal proteins and tighter competition for natural resources forces livestock and aquaculture production to use resources more efficiently. Both sectors have grown and changed very quickly, often outpacing the development of public policies. This process often resulted in poor management of public goods and requires the development of appropriate policies and institutions.

GERBER P., BRUGÈRE C., ANKERS P., 2011. Évolution des productions animales terrestres et aquacoles dans le monde : tendances globales et implications économiques, sociales et environnementales. In : Numéro spécial, Elevage en régions chaudes. Coulon J.B., Lecomte P., Boval M., Perez J.-M. (Eds). INRA Prod. Anim., 24, 9-22. 
